\title{
Energy Provisioning Technique to Balance Energy Depletion and Maximize the Lifetime of Wireless Sensor Networks
}

\author{
Hassan Hamid Ekal ${ }^{1^{*}}$ \\ Department of Communication Engineering, Faculty Of \\ Electrical and Electronic Engineering, University Tun \\ Hussein Onn Malaysia, Johor, Malaysia
}

\author{
Jiwa Bin Abdullah ${ }^{2}$ \\ Department Of Communication Engineering, Faculty Of \\ Electrical and Electronic Engineering, University Tun \\ Hussein Onn Malaysia, Johor, Malaysia
}

\begin{abstract}
With the promising technology of Wireless Sensor Networks (WSNs), lots of applications have been developed for monitoring and tracking in military, commercial, and educational environments. Characteristics of WSNs and resource limitation impose negative impacts on the performance and effectiveness of these applications. Imbalanced energy consumption among sensor nodes can significantly reduce the performance and lifetime of the network. In multi-hop corona WSN, the traffic imbalance among sensor nodes will make nodes located near to the sink consume more energy and finish their energy faster than those distant from the sink. This would cause what is called "energy hole", which prevents the network from performing the intended tasks properly. The objective of the work in this paper is to balance energy consumption to help improving the lifetime of corona-based WSNs. To maximize the lifetime of the network, an innovative energy provisioning technique is proposed for harmonizing the energy consumption among coronas by computing the extra needed energy in every corona. Experimental results of the evaluation revealed that the proposed technique could improve the network lifetime noticeably via fair balancing of energy consumption ratio among coronas.
\end{abstract}

Keywords-Wireless Sensor Network (WSN); Lifetime; Node deployment; Energy provisioning

\section{INTRODUCTION}

The high potential for utilization in numerous applications has made wireless sensor networks (WSNs) widely popular. A lot of applications regarding event and activity measurements have been developed for monitoring and tracking in different environments, where human access is dangerous. These applications include intelligence traffic and supply chain management, health care and habitat monitoring, gas and temperature detection, military and national security domains, and many others [1, 2]. WSNs are considered as dynamic multi-hop routing networks, where sensor nodes are connected to centralized powerful machine, called Base Station (BS) or sink. WSNs characteristics impose serious challenges on the design of WSNs [3]. Due to the size and nature, sensors have restricted power capacity, and thus, they have limits in processing and computing capacities.

In most scenarios, sensor nodes are immobile and do not change location after deployment. However, sensors activities usually result in power dissipation in some regions, which causes a dynamic change in the network topology. Also, sensor nodes are programmed to alternatively be in active mode and in sleeping mode to save energy. In sleeping mode, sensors are disconnected from the network as they turn off their transceivers. With such dynamic changes in the network topology, providing connectivity, and at the same time, minimizing the energy consumption is difficult.

Thus, reliable energy-efficient mechanisms for WSNs can help prolong the lifetime of the network considerably [4]. Figure 1 shows an example of WSN architecture in operation.

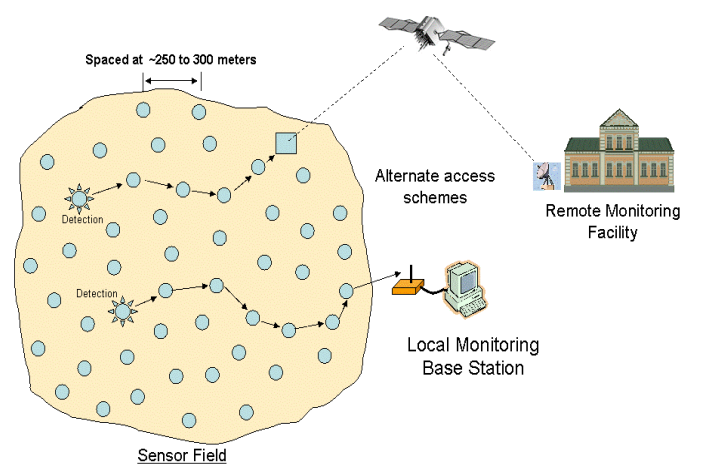

Fig. 1. Example of WSN architecture

With the inherited limitations of sensor nodes, WSNs therefore have networking resource limitation such as communication range and bandwidth, frequent topology changes, high density and node unreliability [5]. In addition to the aforementioned characteristics and limitations, there are several challenges in deploying WSNs effectively. These challenges include scalability, routing, energy consumption, as well as self-organizing and fault tolerance [6].

\section{REQUIREMENTS AND CHALLENGES OF SENSING APPLICATIONS}

Sensor nodes should be capable of managing, controlling and organizing themselves into a network in order to effectively perform their tasks [7]. This would help in facilitating the network management and satisfying the application requirements. However, the limited resources of sensor nodes make it necessary to have reliable 
communication protocols and efficient management services. Improving communications and reducing energy consumption in the network require communications protocols to be implemented at different layers that present the protocol stack.

This can be noticeably operative with regard to network efficiency, as well as latency and energy consumption. Nonetheless, communication protocols developed for traditional networking are not appropriate for WSNs, as they are not projected to operate under such resources limitation. Therefore, numerous energy-efficient protocols have been proposed to operate in all of the five layers in the protocol stack. Communications among the layers is supported by utilizing the concept of cross-layering. In other words, the information of protocol in a certain layer is shared across the rest of the layers, to satisfy some requirements of WSNs [1] [8-13].

As sensor nodes operate with a limited power, their energy usage is very important matter in designing and deploying WSNs. Consequently, much research work has been done on energy harvesting and moderating energy consumption [1421] [23]. Once a sensor node finishes its energy, it will be disconnected from the network; this may have significant effect on the performance of the application. The network lifetime basically relies on the connectivity and number of active nodes in the network. Therefore, energy should be utilized resourcefully in order to make the best use of the lifetime of the network operation. Energy or power harvesting (also known as energy scavenging) is the process where sensor nodes derive their energy from external sources including solar cells [6] [22], fuel cells, wind energy, salinity gradients, mobile supplier, and acoustic noise [23].

These energy source techniques are utilized to overcome the energy limitation and also to provide satisfactory level of quality of service (QoS) to the application. Reliable communications are required to support the data transmission to the intended destination. Thus, buffer monitoring, congestion control and packet-loss recovery mechanisms are needed. Communication strength relies on the sensors placement. Sparse deployment of sensors may result in higher energy usage due to distant transmission. On the other hand, dense deployment of sensors may lead to more local communications due to short-range transmission. Coverage is of vital concern when it comes to sensor deployment. The coverage area of the network is specified by the number of sensors deployed [6].

\section{ENERGY ISSUE IN CORONA-BASED WSNS}

In many-to-one multi-hop network architecture, energy consumption of the sensor nodes is often imbalanced. Nodes placed close to the sink (or BS) certainly consume more energy than those located far from the sink [24], and accordingly, they finish their energy (die) earlier than the distant ones which are unable to send data to the sink even though they have much unused energy. Consequently, energy holes (hot spots) are created in the network [25-27]. In contrast, without multi-hopping, distant sensor nodes have to transmit data to the sink directly. Thus, due to long transmission distance, they finish their energy faster than the ones closer to the sink. The network is partitioned into islands when energy holes phenomenon happens. This will reduce the network lifetime considerably, and the network dies before performing the intended tasks completely. In multi-hop corona WSNs (Figure 2), energy holes occur at the inner coronas (rings) which are close to the sink.

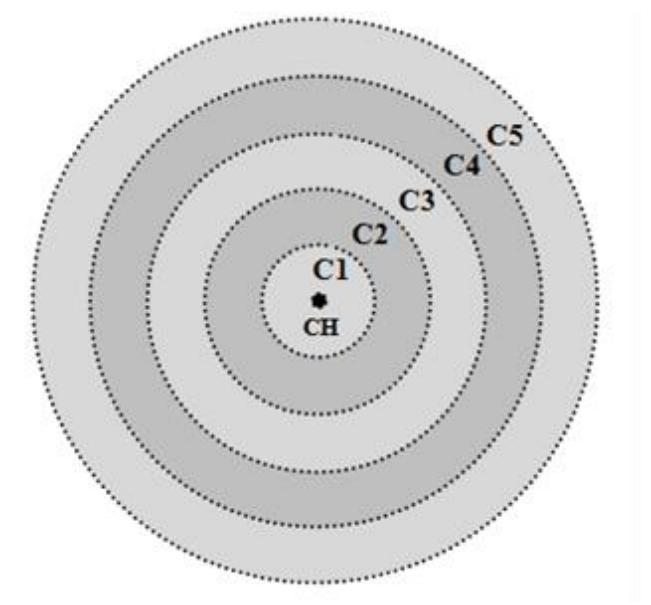

Fig. 2. Many-to-one Multi-hop corona-based WSN

There have been several solutions proposed to alleviate this problem through the use of non-uniform node deployment, dynamic methods (through mobile sink or clustering algorithm), transmission range (control of multilevel ranges), and heterogeneous nodes (nodes with capabilities for relaying data and/or energy provisioning)[2834]. Figure 3 shows some of the solutions, which can be demonstrated by using multi-hop corona network model, to overcome the energy holes problem.

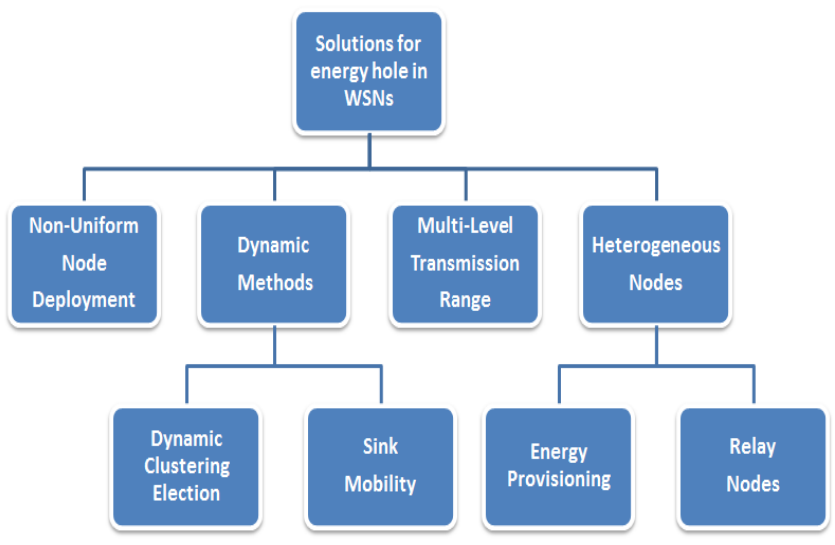

Fig. 3. Solutions developed to overcome energy hole problem

The lifetime of the network can be prolonged by eliminating (or limiting the development of) the energy holes. This can be achieved by designing energy-efficient solutions for the five layers that form WSN, namely physical layer, data link layer, network layer, transport layer, and application layer. In designing those solutions, several issues such as security, storage, synchronization, localization, coverage, data compression and aggregation should be considered. Reliable algorithms of those solutions may improve the network lifetime remarkably.

Improving the lifetime is one of the challenges when 
designing WSNs, as it relies on a number of parameters such as network architecture, protocols, energy model, channel characteristics, techniques for data collection, and also on how the lifetime of the network is defined [35]. Tackling and mitigating the factors (such as area under monitoring, number of coronas, corona width, node deployment strategy, and node transmission range) that bring about or precipitate the energy hole may also enhance the lifetime.

The aim of the research presented in this paper is to propose an energy-balanced provisioning technique to alleviate the energy hole problem in multi-hop corona WSNs to overcome the limitations of the previous research works and provide practicable enhancement to WSNs in terms of network lifetime, energy consumption, and connectivity. The rest of this paper is organized as follows: Section 4 presents the design of the proposed energy provisioning technique. Section 5 describes the simulation scenarios for performance evaluation of the proposed technique and presents the analytical results gained from simulations and the comparison to the traditional uniform technique from energy distribution point of view. Finally, Section 6 concludes this paper and suggests some related future work.

\section{Proposed Energy Provisioning TechniQue}

Energy depletion of the coronas can be balanced by distributing the energy to some extent, but requires the energy in each corona to be identified beforehand. Also, to achieve the maximum network lifetime, it is necessary to consider the additional energy needed to balance the energy consumption between coronas by defining the initial energy of each node with regard to the corona location where the node resides. This can be achieved by using a new energy provisioning technique that is based on innovative mathematical system.

This section presents theoretical design of the proposed energy provisioning technique, which is based on the calculation of the energy increment ratio in each corona, aiming at balancing the energy consumption. For energy provisioning, the mathematical relationship between network lifetime and the additional energy that is necessary to balance energy depletion among coronas will be estimated. The procedure starts by calculating the network lifetime in each corona. Then, the relationship between the energy consumption of each corona and the energy needed to relay packets received from the nodes of the outer coronas is determined. Next, the extra energy required for nodes in each corona to support the transmission of data packets from outer coronas is estimated. After that, the relationship between increasing the network lifetime and the extra energy required is formulated. Here, for energy increment computation, the extra energy required to balance the energy in each corona is computed.

\section{A. Corona model and the corresponding assumptions \& definitions}

The research work presented in this paper considers a circular multi-hop corona WSN with immobile sink placed at the center of the circular area. The network radius is $\operatorname{rad}_{R}$ and each node in the network operates with a transmission range of $R$. As the corona network model can be represented by $(k+1)$-tuple $\left(k, w_{1}, \ldots, w_{k}\right)$, the corona model covering the circular area can be divided into $k$ number of coronas $C$ of a specific width w. $C_{\mathrm{i}}$ indicates the $i$ th corona where $C_{1}$ represents the first corona (innermost corona). $w_{1}$ denotes the radius of the inmost corona, while $w_{i}=2,3, \ldots, k$ signifies the width of any other corona $i$. Corona network model can be categorized into uniform-width and non-uniform-width, based on the corona width/radius. In the uniform-width corona model, $w_{i}=s_{i}$ for all $i=1,2,3, \ldots, k$, meaning that width of every corona is the same as the sensing range of the node. On the other hand, in the non-uniform-width corona model, there is only one corona with a width (or radius) that is different from the rest of coronas, either shorter or longer. In this paper, only the uniform-width corona model was considered to avoid associated complexity and high cost of estimating the width of non-uniform corona model. Every node was to create and transmit $l$ bits per unit of time via the multi-hop communication, through which, sensor nodes which belonged to corona $C_{i}$ would forward incoming data to next corona $\left(C_{i-1} \mid i \geq 2\right)$. The area under monitoring was assumed within an environment with PLE of 4 (that is, $n=4$ ), and the communication environment was considered contented and error-free. The proposed energy provisioning technique was considered for constant monitoring applications only, as event-driven applications are out of the scope of the research. The commonly used q-switch routing algorithm in WSN was utilized in this study, while data aggregation was not considered as it might cause imbalanced energy consumption.

For corona-based WSNs, the following definitions regarding the lifetime are applicable:

Definition 4.1. The lifetime of a corona $C_{i}\left(l C_{i}\right)$ (in unit time) is determined by the ratio:

$$
l C_{i}=\frac{T \varepsilon_{C_{i}}}{E C_{i}}
$$

where $T \varepsilon_{C_{i}}$ is the total initial energy of $C_{i}$, and $E C_{i}$ is the energy consumption in corona $C_{i} . T \varepsilon_{C_{i}}$ can be obtained from $\varepsilon N_{i}$, where $\varepsilon$ represents the node initial energy and $N_{i}$, represents the number of nodes in corona $C_{i}$.

Definition 4.2. The lifetime of the network $l t_{n e t}$ is the total time from the point at which the network starts operating, to the point when the first node finishes its energy (dies). In other words, the lifetime of the network is represented by the lifetime of first node. In case the energy consumption of nodes inside corona $C_{i}$ being uniform, the energy consumption of each node $\left(E N_{i}\right)$ in corona $C_{i}$ is:

$$
E N_{i}=\frac{E C_{i}}{N_{i}}
$$

Accordingly, the lifetime of every node $\left(l t_{N_{i}}\right)$ in corona $C_{i}$ is estimated using: 


$$
l t_{N_{i}}=\frac{\varepsilon N_{i}}{E C_{i}}
$$

The lifetime of each sensor node $\left(l t_{N_{i}}\right)$ should be similar to the lifetime of $C_{i}\left(l C_{i}\right)$ as determined in Definition 4.1. Hence, the lifetime of the network is defined by the shortest lifetime of any corona, as follows:

$$
l t_{n e t}=\min _{C_{i}} \frac{\varepsilon N_{i}}{E C_{i}}, \forall C_{i}
$$

Definition 4.3. For corona model $\left(k, w_{1}, \ldots, w_{k}\right)$ with a sensing range $s_{i}$ of the node for $i=1,2,3, \ldots, k$ in corona $C_{i}$ where $\frac{w_{i}}{2} \leq s_{i} \sqrt{2}\left(\sum_{j=1}^{i} w_{j}\right)$, the maximum corona coverage can be achieved if the sensor nodes are deployed with distance $d_{i}^{d e p}$ from the center of corona $C_{i}$. $d_{i}^{d e p}$ can be computed as follows:

$$
\begin{gathered}
d_{i}^{d e p}=\sqrt{\frac{\left(\sum_{j=1}^{i-1} w_{j}\right)^{2}+\left(\sum_{j=1}^{i} w_{j}\right)^{2}-2 s_{i}^{2}}{2}}, \\
i=2,3, \ldots, k
\end{gathered}
$$

\section{B. Computation of the ratio of energy increment}

This part of the research work aims to compute the amount of the extra energy required in each corona $i$ to balance the energy. This is done by estimating the increment ratio of energy $V$ needed in any corona $C_{i}$, which helps in balancing the energy depletion among the sensor nodes, based on the geometric area of coronas in the area of monitoring.

To relay a packet from outer coronas towards the sink, each sensor node located in corona $i$ would need $E_{N_{i}}$ amount of energy, which can be obtained as in Equation (5) below:

$$
E_{S_{i}}=\left(\mathrm{E}_{\mathrm{rx}}+\mathrm{E}_{\mathrm{tx}}\right) / \mathrm{E}_{\mathrm{tx}}
$$

where $E_{t x}$ denotes the energy needed to transmit one bit of data over a definite distance $d$, which can be computed as in Equation (6) below:

$$
E_{t x}=E_{\text {elec }}+\alpha d^{n}
$$

where $\alpha$ denotes the energy depletion over the operational amplifier throughout the data transmission, $d$ which indicates the transmitter-receiver distance, and $n$ indicates the Path Loss Exponent (PLE) depending on the system environment.

$E_{r x}$ in Equation (5) denotes the energy needed for data reception, which can be achieved as in Equation (7).

$$
E_{r x}=E_{\text {elec }}
$$

where $E_{\text {elec }}$ represents the electronic energy. Accordingly, if by letting $\mathbb{R}^{(i+1), k}$ to denote the region covered from corona $(i+1)$ to corona $k$ (i.e. $[(i+1), k]=\left\{C_{i} \in \mathbb{R}^{(i+1), k} \mid i+1 \leq\right.$ $\left.C_{i} \leq k\right\}$, and $\mathbb{R}_{C}^{i}$ to denote the region of corona $C_{i}$, then the increment ratio of energy of any corona $C_{i}$ for conveying the packets from outer coronas to the innermost corona is estimated as in Equation (8):

$$
V_{i}=\frac{\mathbb{R}^{(i+1), k}}{\mathbb{R}_{C}^{i}} \times E_{N_{i}}
$$

From Equation (8), Equation (9) is derived as:

$$
V_{i}=\frac{\int_{0}^{2 \pi} \int_{i \times \mathrm{w}}^{r a d} \mathrm{r} d \mathrm{r} d \theta}{\int_{0}^{2 \pi} \int_{(i-1) \times w}^{i \times w} \mathrm{r} d \mathrm{r} d \theta} \times E_{N_{i}}
$$

Further simplification yields Equation (10) as follows:

$$
V_{i}=\frac{\operatorname{rad}_{R}^{2}-i^{2} w^{2}}{2 i w^{2}-\mathrm{w}^{2}} \times \frac{\mathrm{E}_{\mathrm{rx}}+\mathrm{E}_{\mathrm{tx}}}{\mathrm{E}_{\mathrm{tx}}}
$$

where $\operatorname{rad}_{R}, w, E_{r x}$, and $E_{t x}$ denote the radius of the network, width of each corona, reception energy and transmission energy of one bit, respectively. By letting $\operatorname{rad}_{R}=k \times w$, then the increment ratio of energy of corona $C_{i}$ needed to achieve energy balancing is calculated as:

$$
V_{i}=\frac{k^{2}-i^{2}}{2 i-1} \times E_{N_{i}}
$$

In this case, the packets generated in the external coronas should be relayed to the inner ones via corona $C_{i}$, thus, the amount of energy needed in corona $C_{i}$ in order to relay the received packets must be supplied to corona $C_{i}$.

\section{Sensing, relaying, and idle scheduling}

To achieve improved efficiency of the network, it is assumed that each sensor node can be in one of two sates, either in the sensing state or in the idle state. Sensor nodes which are selected to sense the monitoring area can perform sensing only or along with data relaying, whereas those not selected for sensing may remain idle or relay data received from other coronas.

Energy consumption of a sensor node can be controlled by proper scheduling between the sensing and relaying operations or move to idle state, by utilizing efficient and simple turnbased scheduling algorithm. Counter (count) is used to regulate whether a sensor node should sense/relay by that time or not. The algorithm is presented in Figure 4. 
/*as the sensor node still has energy, it is enabled to sense the monitoring area* [1] whlie (nodeEnergy $>0$ )

[2]

$$
\text { Sense }=\text { true }
$$

[3] if $\left(\right.$ count $_{i} \bmod$ count $\left._{i}^{\max }=0\right)$ then

$/ *$ the sensor node is enabled to sense and also relay data from other corona $C_{i}^{*}$; if $($ Relay $=$ true $)$ then

Send(ownData + relayData);

else

* the sensor node sends its own data only*/ Send(ownData)

end if

[9] else if $\left(\right.$ count $\left._{i} \bmod \operatorname{count}_{i}^{\max } \neq 0\right)$ then

/* the sensor node is not enabled for sensing, but enabled to relay data*/ if $($ Relay $=$ true $)$ then

$[10]$

$[11]$

[12]

[13] Send(relayData)

if

* Change the count value for the sensor node*;

[14] count $_{i}=\left(\right.$ count $\left._{i}+1\right) \bmod$ count $_{i}^{\max }$;

[15] end whlie

Fig. 4. Pseudo-code of the scheduling algorithm

While nodes in corona $C_{i}(i=1,2,3, \ldots, k)$ are placed with uniform space of $2 \pi d_{i}^{d e p} / N_{i}$ to each other, sensor nodes in corona $C_{1}$ are placed in the middle of the corona width (at point $w_{1} / 2$ )with uniform space of $\pi w_{1} / N_{1}$ to each other.

Before being in either state, sensor nodes should be assigned a to count $_{i}$ value that varies from 1 to count $_{i}^{\max }=$ $N_{i} / N_{i}^{\text {min }}$, where $N_{i}^{\text {min }}$ is the minimum number of deployed nodes that can cover corona $C_{i}$. The count value of count $_{i}^{\max }$ is assigned to the first sensor node and the value count $_{i}^{\max }-1$ is assigned to the following sensor node. This procedure is repeated until count of value 1 is reached, and until all nodes are assigned with count values.

Nodes operate in rounds, where they perform sensing, transmitting, and receiving data in a fixed time interval. When the network operates, the sensor node starts sensing (and might forward data as well) if it's count $_{i} \bmod \operatorname{count}_{i}^{\max }=0$. In the subsequent round, count value will be increased by 1 for every sensor node in the network. This is done in a cyclic routine to define which node should sense the monitoring area and relay data; if the current value for count is $\operatorname{count}_{i}^{\max }$, the next value for count will be 1 , and so on.

\section{PERFORMANCE EVALUATION}

To validate the proposed energy provisioning technique aimed at balancing energy consumption and maximizing the lifetime in WSNs, evaluation scenarios (described in the following subsection) had been simulated using MATHLAB on a machine running the CentOS 5.5 version of the Linux operating system.

\section{A. Evaluation scenarios}

The corresponding parameters setup for the evaluation scenarios of the proposed technique is presented in Table 1.
TABLE I. SCENARIO PARAMETERS SETUP

\begin{tabular}{|l|c|}
\hline \multicolumn{1}{|c|}{ Parameter } & Value \\
\hline \hline Initial energy of each node $\varepsilon$ & $0.5 \mathrm{~J}$ \\
\hline Electronic energy consumption $E_{\text {elec }}$ & $50 \mathrm{~nJ} / \mathrm{bit}$ \\
\hline Network radius $\operatorname{rad}_{R}$ & $500 \mathrm{~m}$ \\
\hline Geometric progression $q$ & 2 \\
\hline Energy dissipate $\alpha$ & $0.0013 \mathrm{pJ} / \mathrm{bit} / \mathrm{m}^{4}$ \\
\hline Packet length $l$ & $300 \mathrm{bits}$ \\
\hline Path loss exponent $n$ & 4 \\
\hline Number of coronas $k$ & 10 \\
\hline Uniform corona width $w$ & $100 \mathrm{~m}$ \\
\hline Transmission range $R$ & $100 \mathrm{~m}$ \\
\hline
\end{tabular}

For the evaluation scenarios, the sensor transmission range was made equal to the sensing range and also to the width of the corona it resided in. Having a corona network with radius $\operatorname{rad}_{R}$, the total number of coronas $k$ was achieved by using:

$$
k=\frac{\operatorname{rad}_{R}}{R}
$$

That is, for a given network radius $\operatorname{rad}_{R}$ of $500 \mathrm{~m}$ and Transmission range $R$ of $100 \mathrm{~m}$, the number of coronas $k$ should be 5 .With each round of $1 \mathrm{~ms}$ period, every sensing node would generate data of 300 bits.

For comparison purpose, the minimum number of sensor nodes $N_{i}{ }^{\min }$ for covering a uniform $\operatorname{corona} C_{i}$ width $w$ of 100 was set to $12,36,64,84$, and 120 , for $N_{1}, N_{2}, N_{3}, N_{4}, N_{5}$, respectively, based on Cosine Rule. The performance experiments of the evaluation scenarios had been conducted 20 times as the minimum test requirement in computer sciences to achieve non-overlapping confidence interval. The evaluation experiments were done for both the energy distribution based on the proposed technique and normal (uniform) energy distribution under similar parameters setup described earlier.

\section{B. Experimental results}

Figure 5 shows the ratio of energy needed to be added to each of the four coronas $C_{1}, C_{2}, C_{3}, C_{4}$ (as $C_{4}$ was the last corona and its sensor nodes did not relay data from other coronas) in order to improve the network lifetime with balanced energy consumption. From the figure, to maximize the lifetime of a network of radius $\mathrm{rad}_{R}$ of $500 \mathrm{~m}$ where the width of each corona was $100 \mathrm{~m}$, the ratios of required additional energy to the corresponding coronas $C_{1}, C_{2}, C_{3}, C_{4}$ were approximately $65.50,21.40,12.19$ and 9.00 times more than the normal (uniform) case without the use of the proposed technique. 


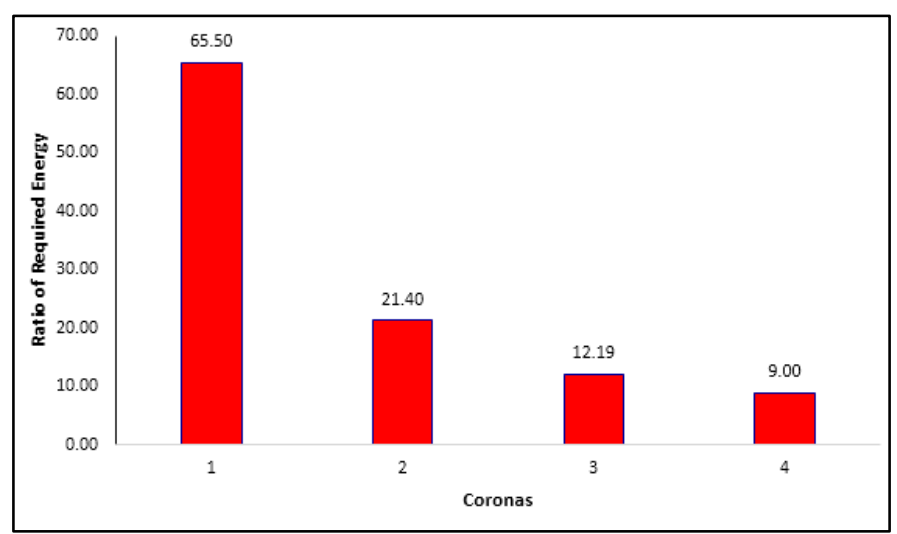

Fig. 5. Ratio of energy increment in each corona for balanced energy consumption

With the evaluation of the proposed technique by increasing the energy in the network and distributing the extra energy in the coronas accordingly, the results also showed that the network lifetime had been increased considerably. Table 2 presents the percentage of the lifetime increase for coronas $C_{1}$, $C_{2}, C_{3}$ and $C_{4}$ when the total network energy was increased using the proposed technique compared to the use of normal (uniform) energy distribution.

TABLE II. LIFETIME RATIO GAINED THROUGH INCREASING ENERGY USING THE PROPOSED TECHNIQUE VS. NORMAL ENERGY DISTRIBUTION

\begin{tabular}{|c|c|c|c|c|c|}
\hline 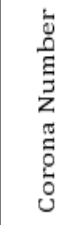 & 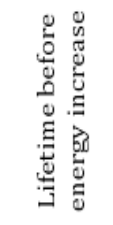 & 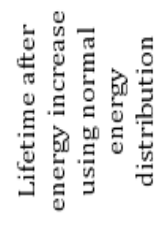 &  & 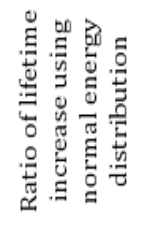 & 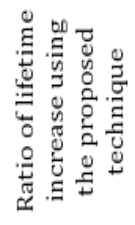 \\
\hline 1 & 192.3005 & 384.6 & 384. & 2 & 2 \\
\hline 2 & 768.405 & \begin{tabular}{|l|}
1536.815 \\
\end{tabular} & 3513.395 & 2 & 4.6 \\
\hline 3 & 1720.645 & 3441.29 & 11846.65 & 2 & 6.9 \\
\hline 4 & 3019.33 & 603 & 28126.65 & 2 & 9.4 \\
\hline
\end{tabular}

From the data as shown in Table 2, it is clear that, with the use of the proposed technique, the total lifetime could be increased $9.4\left(>2^{3}\right)$ times, whereas it could be increased only by $2\left(=2^{0}\right)$ times when using normal energy distribution. It was found that, by increasing the total energy according to derived Equation (11) in the proposed energy provisioning technique, the network lifetime could be increased remarkably. The results showed that the lifetime had been improved by about $40 \%$ when using the proposed technique compared to the uniform technique for energy distribution.

\section{CONCLUSION AND FUTURE WORK}

In multi-hop corona Wireless Sensor Networks (WSNs) with uniformly distributed nodes, when an energy hole appears due to the death of some nodes in critical location, data packets cannot be sent from distant nodes to the sink. This means that the network lifetime finishes early, resulting in wastage of a significant amount of energy. This paper presents the theoretical design and development of an innovative energy provisioning technique to balance energy depletion and maximize network lifetime. The experimental evaluation results reveal that, when using the proposed technique for efficient energy distribution by computing the extra needed energy, the lifetime can be enhanced by about $40 \%$ compared to that of the uniform technique. With the proposed technique, the network can be considered homogeneous if the sensor nodes capabilities are the same, and that the extra added (required) energy can be provided by adding more sensors to meet the total energy requirements in specific coronas. Otherwise, sensor nodes in those coronas could be supplied by different initial energy to fulfill the energy needed. The network in such case is heterogeneous, where defining the initial energy in every node with regard to its corona can be beneficial to enable a proper utilization of the proposed technique in heterogeneous WSN. This objective is considered in the future work.

\section{ACKNOWLEDGEMENT}

The authors would like to thank the management and the Centre For Graduate Studies of University Tun Hussein Onn Malaysia (UTHM) for sponsoring the research. The authors would like also to appreciate the assistance from the Office for Research, Innovation, Commercialization and Consultancy Management (ORICC).

\section{REFERENCES}

[1] Holger Karl, A. W., Protocols and architectures for wireless sensor networks, Book, ISBN: 0-470-09510-5, 2005.

[2] Shi Lan; Miao Qilong; Jinglin Du, "Architecture of Wireless Sensor Networks for Environmental Monitoring," Education Technology and Training, 2008. and 2008 International Workshop on Geoscience and Remote Sensing. ETT and GRS 2008, vol.1, no., pp.579, 582, 21-22 Dec. 2008

[3] Islam, A. K M M; Baharun, S.; Wada, K., "An overview on Dynamic Wireless Sensor Network Architectures," Informatics, Electronics \& Vision (ICIEV), 2012 International Conference on, vol., no., pp.464,468, 18-19 May 2012.

[4] Akyildiz, I. F., Wireless Sensor Networks, Series in Communications and Networking. John Wiley amp; Sons Ltd., 2010.

[5] Houda Labiod, Wireless Ad Hoc and Sensor Networks ISTE Ltd, John Wiley and Sons, Inc. ISTE Ltd, 2008

[6] Yick, J., Mukherjee, B., \& Ghosal, D., Wireless sensor network survey. Computer Networks, 52, 2292-2330, 2008.

[7] Jiong Jin; Yee Wei Law; Wei-Hua Wang; Palaniswami, M., "A hierarchical transport architecture for wireless sensor networks," International Conference on Intelligent Sensors, Sensor Networks and Information Processing, 2008. ISSNIP 2008, vol., no., pp.145,150, 1518 Dec. 2008

[8] Berfield, A. \& Mosse, D., (2006), Efficient Scheduling for Sensor Networks, 3rd Annual International Conference on Mobile and Ubiquitous Systems - Workshops, 2006.

[9] Berfield A., Chrysanthis P.K \& Labrinidis A., (2006), Efficient handling of sensor failures, Proceedings of the 3rd workshop on Data management for sensor networks: in conjunction with VLDB 2006, ACM.

[10] Zabin, F., Misra, S., Woungang, I. , Rashvand, H.F., Ma, N.-W. \& Ali, M. A., (2008), REEP: data-centric, energy-efficient and reliable routing protocol for wireless sensor networks, Communications, IET, pages $995-1008$.

[11] Casari, P. , Nati, M. ; Petrioli, C. \& Zorzi, M. (2007), Efficient NonPlanar Routing around Dead Ends in Sparse Topologies using Random Forwarding, ICC '07. IEEE International Conference on Communications, Pages $3122-3129$.

[12] Chamam A. \& Pierre S., (2009), A distributed energy-efficient clustering protocol for wireless sensor networks, Computers \& Electrical Engineering, Volume 36, Issue 2, March 2010, Pages 303-312 
[13] Baghyalakshmi, D., Ebenezer, J. \& Satyamurty, S.A.V., (2010), Low latency and energy efficient routing protocols for wireless sensor networks, International Conference on Wireless Communication and Sensor Computing, ICWCSC 2010. Pages $1-6$.

[14] Azad, A. K. M., \& Kamruzzaman, J. (2011). Energy-Balanced Transmission Policies for Wireless Sensor Networks. Mobile Computing, IEEE Transactions on, 10, 927-940.

[15] Ammari, H. M., \& Das, S. K., Promoting Heterogeneity, Mobility, and Energy-Aware Voronoi Diagram in Wireless Sensor Networks. IEEE Transactions on Parallel and Distributed Systems, 19, 995-1008, 2008.

[16] Charilaos, E., Sotiris, N., \& Jose, R. (2006). Energy balanced data propagation in wireless sensor networks. Wirel. Netw., 12, 691-707.

[17] Chen, Y., Li, Q., Fei, L., \& Gao, Q. (2012, 9-12 Sept. 2012). Mitigating energy holes in wireless sensor networks using cooperative communication. Paper presented at the Personal Indoor and Mobile Radio Communications (PIMRC).

[18] Cheng, P., Chuah, C. N., \& Liu, X. (2004). Energy-aware node placement in wireless sensor networks. Paper presented at the Global Telecommunications Conference, 2004, GLOBECOM'04.

[19] Guo, W., Liu, Z., \& Wu, G. (2003). An energy-balanced transmission scheme for sensor networks. Paper presented at the 1st international conference on Embedded Networked Sensor Systems.

[20] Haibo, Z., \& Hong, S., Balancing Energy Consumption to Maximize Network Lifetime in Data-Gathering Sensor Networks, IEEE Transactions on Parallel and Distributed Systems, 20, 1526-1539, 2009.

[21] Esseghir, M., Bouabdallah, N., \& Pujolle, G. (2007). Energy provisioning model for maximizing wireless sensor network lifetime. Paper presented at the Global Information Infrastructure Symposium, GIIS 2007.

[22] Raghunathan, V., Kansai, A., Hse, J., Friedman, J., \& Srivastava, M. (2005). Design considerations for solar energy harvesting wireless embedded systems. Information Processing in Sensor Networks, IPSN $2005,1,457-462$.

[23] Rahimi, M.; Shah, H.; Sukhatme, G.; Heideman, J.; Estrin, D., "Studying the feasibility of energy harvesting in a mobile sensor network," IEEE International Conference on Robotics and Automation, ICRA '03, vol.1, no., pp.19,24 vol.1, 14-19 Sept. 2003.

[24] Ramos, H.S.; Oliveira, E.M.R.; Boukerche, A.; Frery, A.C.; Loureiro, A.A.F., "Characterization and mitigation of the energy hole problem of many-to-one communication in Wireless Sensor Networks," International Conference on Computing, Networking and Communications (ICNC), vol., no., pp.954,958, Jan. 30 2012-Feb. 2 2012.
[25] Perillo, M., Cheng, Z., \& Heinzelman, W. (2005, 17-21 July 2005). An analysis of strategies for mitigating the sensor network hot spot problem. Paper presented at the Mobile and Ubiquitous Systems: Networking and Services, MobiQuitous 2005.

[26] Meicheng, L., Jie, Z., Ming, L., Yuming, B., "A novel solution for energy hole of Wireless Sensor Network," 33rd Chinese Control Conference (CCC), vol., no., pp.456,460, 28-30 July 2014

[27] Pathak, A.; Zaheeruddin, Z.; Tiwari, M.K., "Minimizing the Energy Hole Problem in Wireless Sensor Networks by Normal Distribution of Nodes and Relaying Range Regulation," Fourth International Conference on Computational Intelligence and Communication Networks (CICN), vol., no., pp.154,157, 3-5 Nov. 2012

[28] Nazir, B.; Hasbullah, H., "Mobile Sink based Routing Protocol (MSRP) for Prolonging Network Lifetime in Clustered Wireless Sensor Network," International Conference on Computer Applications and Industrial Electronics (ICCAIE), vol., no., pp.624,629, 5-8 Dec. 2010

[29] Xiaobing, W., Guihai, C., \& Das, S. K., Avoiding Energy Holes in Wireless Sensor Networks with Nonuniform Node Distribution. IEEE Transactions on Parallel and Distributed Systems, 19, 710-720, 2008.

[30] Qian Zhao; Nakamoto, Y., "Routing Algorithms for Preventing Energy Holes and Improving Fault Tolerance in Wireless Sensor Networks," Computing and Networking (CANDAR), 2014 Second International Symposium on , vol., no., pp.278,283, 10-12 Dec. 2014.

[31] Yu Xue; Xiangmao Chang; Shuiming Zhong; Yi Zhuang, "An efficient energy hole alleviating algorithm for wireless sensor networks," Consumer Electronics, IEEE Transactions on, vol.60, no.3, pp.347,355, Aug. 2014.

[32] Liu Meicheng; Zhang Jie; Lyu Ming; Bo Yuming, "A novel solution for energy hole of Wireless Sensor Network," Control Conference (CCC), 2014 33rd Chinese, vol., no., pp.456,460, 28-30 July 2014.

[33] Saleem, F.; Javaid, N.; Moeen, Y.; Akbar, M.; Khan, Z.A.; Qasim, U., "MEET: Multi-hop Energy Efficient Protocol for Energy Hole Avoidance Using Variable Transmission Range in Wireless Sensor Networks," Broadband and Wireless Computing, Communication and Applications (BWCCA), 2014 Ninth International Conference on, vol., no., pp.478,484, 8-10 Nov. 2014.

[34] Kim, Min-Gon; Young-Tae Han; Hong-Shik Park, "Energy-Aware Hybrid Data Aggregation Mechanism Considering the Energy Hole Problem in Asynchronous MAC-Based WSNs," Communications Letters, IEEE, vol.15, no.11, pp.1169,1171, November 2011.

[35] Chen, Y., \& Zhao, Q. (2005). On the lifetime of wireless sensor networks. Communications Letters, IEEE, 9, 976-978. 